and indeed it may be doubted whether, commercially considered, it might not have been wise to have divided the work into two or more independent volumes. Taken, moreover, as it is presented to us, it may perhaps further be affirmed that this would have been a gain to the reader, since the first part comprises the elementary topics of the principles of physical diagnosis, and their application to the investigation of diseases of the chest-topics which must of necessity be subsequently more or less iterated in the application of these to the study of the several forms of thoracic disease. Although, therefore, we think that both student and practitioner would have had cause to rejoice in some measure of abridgment or condensation, we can safely commend this work to their attention, as conveying a vast amount of scientific information, and as furnishing a full and safe practical guide in treatment.

\title{
Review III.
}

1. Contributions to Practical Medicine. By Jasres BegBie, M.D., F.R.S.E., \&c.-Edinburgh, 1862 . pp. 318.

2. Clinical Medicine: Observations recorded at the Bedside, with Commentaries. By W.T. GAIrdner, M.D.-Edinburgh, 1862. pp. 741.

AIx who regard the progress of medicine with an intelligent and friendly eye, must have witnessed with sincere gratification the prodigious advance that has taken place in recent times in microscopical anatomy and in physiological chernistry. That the inquiries into these subjects which have been so ardently pursued, are steps in the right direction we most willingly admit. But we cannot help thinking that the zeal with which these fascinating subjects have been prosecuted, has led us too much to regard them as an end, and in some measure to lose sight of the great truth, that in our character of physicians the ultimate abject which we should never lose sight of in any of our investigations, ought to be the extension and improvement of the resources of medicine as a practical art, which has in view the relief of human suffering and the prolongation of human life. It is scarcely possible to deny that some years ago this tendency was discoverable in certain departments of medical literature to such an extent, that it seemed as if medicine were cultivated much more with reference to its biological relations than for any practical results. The evils of such views were neither few nor small. Nor were they confined altogether to the ranks of the profession; they had an influence on the general public anything but favourable to the credit and position of medical men. How this came to pass it might, we think, neither be useless to inquire, nor difficult to explain. At present, however, we content ourselves with merely adverting to the subject, and congratulating ourselves that a more sedate and healthy tone of medical investigation is again asserting itself- $a$ fact, of which the appearance of such works as Dr. Begbie's and Dr. Gairdner's affords most agreeable and satisfactory evidence.

The names of the two authors whose works stand at the head of this Article, are a sufficient guarantee that we do not intend, in these few 
remarks, to recommend either a blind adherence to antiquated opinions, which the rigorous scrutiny of modern investigation may have proved to be erroneous, or the practice of an empirical routine, unsupported by any stable or accredited principles. Both these writers are men of too high standing and too extensive information in the history of our profession and in the recent progress of scientific inquiries, for any one to suppose that what they write tends to exemplify or encourage such notions. Dr. Beglie is a physician whose large experience ranges over a long series of years, and whose great sagacity and judgment give the highest value to the observations which, in his present volume, he has given to the profession. Dr. Gairdner is a man of high accomplishments, of extensive information, and has deservedly a high reputation for independent and philosophical habits of thought. No retrogressive tendencies need be feared in the writings of two such men as these. What we admire in the writings of both, especially Dr. Gairdner, is the sobriety and caution of their views and deductions. Perfectly familiar, as both of them evidently are, with all the facts that tend to support the humoral pathology now so much in favour, they do not lay a greater stress upon these facts than they are legitimately capable of bearing. They do not represent the knowledge of the numerous chemical actions and transformations that go on in the animal economy, as affording either a solution of the great mystery of life, or a complete account of the whole phenomena of disease. Full importance is assigned to them as a series of sequences of great value and interest in certain morbid and certain restorative processes. But they are treated as nothing more, nor are they exalted beyond their just value; to the exclusion of what Lord Bacon calls those "middle principles" which lie between the domain of established but isolated facts, and those higher generalizations after which the human mind ever aspires, but which in many branches of science, as well as our own, are still dimly seen or vaguely guessed at, if not altogether unrevealed.

Having thus shortly characterized the two interesting works which we have classed together, we proceed to give such an account of them individually as our space enables us to offer, beginning with that of Dr. Begbie.

The Papers which form the present volume, the author informs us, have all appeared in the periodicals of the day, and are now collected and reprinted with such additions or corrections as subsequent experience has suggested to him. The first paper treats of gout and the gouty diathesis, and will richly repay a very careful perusal. Most, if not all, practitioners are probably familiar with the symptoms of a fit of regular gout, and also, we hope, with the minute and graphic description given of it by Sydenham. But even in this its most perfect and typical form, it ought, according to modern investigation, to be regarded as the local manifestation of a certain depraved or vitiated state of the constitution, called by medical men a gouty diathesis, which, we need hardly say, may produce numerous and multiform derangements both of function and structure in every organ and texture of the frame, complicating and modifying almost every diseased 
process to which these organs and tissues are liable, even though the person suffering these has never experienced a regular fit of the gout. Dr. Begbie tells us it is not his design to delineate the gouty diathesis, and this, considering the importance and difficulty of the subject, and the qualifications he has shown for the task, we deeply regret. With a candour and uprightness all the more praiseworthy that they are not always met with, even in quarter's where we might expect to find them, Dr. Begbie has narrated his unsuccessful, as well as his fortunate cases. They are told clearly, succinctly, and without the omission of any particulars necessary for their full comprehension. But after reading them very attentively, we feel how desirable it would have been if the author had described the various signs or symptoms by which he has been able to distinguish that constitutional taint which, wherever it exists, is capable of so modifying local inflammation that it ceases to be amenable to those laws which regulate its ordinary progress. We do not find much to guide us in this respect, except that the connexion which Dr. Begbie has shown to exist betwixt the gouty tendency and inflammation of any organ whatever, imposes on us the necessity of rigorous inquiry, to ascertain. whether the sufferer himself, or any of his relatives, have ever shown. symptoms of gout, an inquiry all the more needful if the disease: prove obstinately untractable under the use of remedies usually successful.

The cases detailed by Dr. Begbie, we think, establish the modifying: influence of gout over diseases of the nervous centres and of the nerves. issuing from them-of the circulating system-of the organs of sense -of the digestive apparatus - and of the synovial, mucous, and serous membranes. We cannot condense these cases, but the following are the chief conclusions the author deduces from them, conclusions which it will be seen coincide with those arrived at by other workers in the same field.

Gout is a constitutional disease, sometimes hereditary, sometimes acquired, dependent on the circulation of a materies morbi produced by mal-assimilation. The gouty diathesis is intimately related to the existence of uric acid and its compounds in the system. There are various stages of this diathesis differing in duration and in type, depending, Dr. Begbie thinks, on a poison circulating in the blood. That the primary effects of this are sometimes slow disturbance of functions, chiefly those of the digestive organs and the nervous centres, sometimes active disease, in certain tissues especially, the synovial, serous, and mucous membranes. That the occurrence of active gouts appears to be useful for a time by eliminating the morbid material from the system. That the contaminated blood eventually weakens the tone of the heart and bloodvessels, producing dilatation and attenuation of their cavities and textures, thus leading to congestion and obstruction, and all their consequences, and finally to structural disease from earthy deposit in the circulating apparatus, thus connecting gout with cerebral disease on the one hand, and severe and fatal cardiac affections on the other. While we willingly acknowledge 
the cautious and moderate way in which the author states his views respecting the order of succession in which the phenomena present themselves; still we must admit that much remains to be done before we can be said to have arrived at a knowledge of the primum mobile of the disease. We have not space to compare the old Cullenian pathology with modern views, but we cannot help being struck with the absence of reference to the nerrous system in Dr. Begbie's attempts to indicate the causation of the disease. We can do no more than throw out this suggestion on the subject, and with a brief account of Dr. Begbie's remedial views, we take leave of this instructive and interesting paper. Perfect temperance, active exercise, and care, especially, of the functions of the skin, is looked upon by our author, as by other's, as the basis of preventive treatment. While during the fit, after clearing the prime vice, colchicum wine and the alkaline diuretics, especially the salts of potash, the former in small doses perseveringly used, are what he chiefly confides in. The same remedies he advises in all internal inflammations, indeed in almost all diseased conditions of any organ, where an association of the disease with a gouty tendency can be traced or suspected, either from the history of the patient, or from the anomalous character and unwonted obstinacy of the symptoms under ordinary treatment. This we take to be the main practical lesson of the paper; remembering, however, that the depurants which the author advises nust often be combined with such means as each special class of cases may require. He also places great reliance on cod liver oil as a means of combating every form of gouty cachexia, and improving, as far as they can be improved, the nodosities which are some of the most distressing of gouty sequelæ.

Following the paper on gout, there are two on the connexion between rheumatism and chorea, and of the first-named disease with erythema nodosum; both are interesting, the latter especially; but we cannot now analyze them. We think Dr. Begbie successful in both cases in. - tracing the association which he seeks to establish, especially in the case of rheumatism and erythema nodosum, a striking instance of which lately came under our own observation, in a healthy woman of fifty, worn out by anxiety and long watching. There is a marked discrepancy between the treatment of rheumatism spoken of by Dr. Begbie, with strong approval, at p. 87 , which regards venesection with calomel and opium as the chief therapeutic agents, and the depuratory mode of management by the salts of potash, which he represents at p. 93 as that which he ordinarily employs, owing, we presume, to his adoption of the modern humoral pathology. At another part of the volume he seems still to have recourse occasionally to bloodletting as at least an auxiliary. We could have wished for a little more definite information from one so well qualified to give it, as to his practical views. Certainly there are not many cases of rheumatism more successful than the first case recorded by Dr. Begbie in the Paper on Rheumatism and Chorea, where the treatment was strictly antiphlogistic, and the patient was convalescent in little more than a week. There is a remark in Dr. Begbie's book to the effect that the 
plausibility of the views of the Solidists led us long to shut our eyes to the state of the fluids, especially the blood. It is most true and just, and serious error was the result. But the human mind is prone to extremes. And human progress, in everything but the exact sciences, resembles more a series of oscillations in which the tendency to reach a certain terminus may be clearly observed, than a steady and unfaltering advance. Let us beware, then, that a too exclusive devotion to animal chemistry, or any one subject, does not lead us astray in the opposite direction. The history of medicine proves that the reverse of error is not always or necessarily truth.

The paper on anæmia is so good that we can only commend it to careful study. In the next, on the oxalic diathesis, we do not find much to detain us. That on fatty degeneration of the heart, consists mostly of a narrative of the cases of Dr. Chalmers and Dr. Abercrombie; and that on Diphtheria is so recent, and we believe so well known and highly estimated, that we may safely pass it over. The papers on Stramonium and Arsenic deserve great consideration, not so much on account of what they contain, as from their bringing emphatically before us the testimony of a very competent and trustworthy observer to the value of strictly remedial medication in certain cases at least. The tendency of medical thought has recently been to make general hygienic considerations every thing, and remedies, properly so-called, nothing, or next to nothing. We have therefore great pleasure in listening to Dr. Begbie's exposition of opinions very opposite to this view-itself a reaction from previous exaggeration-so far as regards the use of arsenic and the extract of datura stramonium. The latter given in doses of onethird of a grain three times a day, Dr. Begbie regardsas a most valuable nareotic and antispasmodic, superio" to Dr. Hunt's favourite remedy, belladonna, in many cases of neuralgia. In the former, his confidence is very great, in chorea, in skin diseases, in obscure diseases of the uterine system, and wherever a rheumatic taint may be traced, or suspected, given very steadily till slight inflammation of the eyelids, and a very thin, silvery film on the tongue, shows the physiological action of the drug, and then in smaller doses cautiously continued for a long time.

Dr. Begbie is a strenuous humoralist, and we do not quarrel with him on this score; but we beg to remark that, even when principles well ascertained are applied to subjects where we do not know the whole of the case, the reasoning becomes hypothetical, because what is assumed to make it applicable is hypothesis. What mischief has resulted to our science and our art from the adoption of mere hypotheses, and the more subtle form of hypothetical reasoning we have alluded to, we apprehend few men in the profession know better than Dr. Begbie himself.

The work of Dr. Gairdner, which we now proceed to notice, is characterized by different qualities from those displayed in the volume of Dr. Begbie. And without making any invidious comparison, we have no scruple in saying, it is not inferior either in respect of interest 
or of intrinsic merit. Throughout, it bears the marks of careful observation and scrupulous fidelity, as well as such a readiness and skill in illustration as must place the author very high in the rank of a clinical teacher. But what is perhaps most commendable in the book, is the homage it everywhere pays to the supremacy of truth-truth in matters of fact, and an unswerving desire to ascertain the truth also in matters of speculation. A quality this, inestimably important to the philosophical character of a writer, as well as to his moral status, and which, without being obtrusively or ostentatiously displayed, is unmistakably exhibited in every statement which Dr. Gairdner puts forth, and in every deduction he draws from his facts. We must not spend time, however, in preliminary dissertation, but, so far as we are able, proceed to give an account of the book, or at least of such portions of it as we can select for observation or analysis.

The first papers which we shall notice are those on pneumonia, at the commencement of the volume; and we shall do little more than abridge the summary Dr. Gairdner gives of the principles which regulate the treatment of this disease. Blood-letting is a remedy of great power in cases adapted for its use-that is, in its very early stages -in vigorous constitutions, and when the attending fever is of an inflammatory type, and the symptoms urgent; without attention to these conditions, the practice is sure to pass into a dangerous routine. Hence the extreme difficulty of obtaining comparative statistics respecting the treatment of the disease, for if selected cases only are bled, they cannot be set against unselected cases, or against cases selected for their adaptation to an opposite mode of treatment, and without selection, the application of depleting measures is vicious $a b$ initio as a basis of reasoning, and morally wrong. The statistics of Louis and Grisolle, however, show the power of bleeding to modify the progress and abridge the duration of the disease in cases adapted for its use. Active remedies in pneumonia must be regulated by symptoms, not by physical signs, for the modern idea of the malady includes many cases not recognised as such in former times, and differing essentially in the pathological state of the constitution, from the pneumonia of Cullen, characterized by high synovial fever and urgent pain and dyspnœa-a class of symptoms now very rarely witnessed, and still, when seen, Dr. Gairdner thinks, indicating antiphlogistic remedies. Lastly, we are reminded of the necessity in all cases of bearing in mind the efforts of the tendency to a spontaneous recovery. We ourselves concur entirely in these views, and we believe they will commend themselves to the good sense and sober thinking of the experienced members of our profession.

The papers on pneumonia are followed by two on alcoholic stimulants, which are remarkable for their good sense and moderation. We agree in Dr. Gairdner's view, that with every feeling of respect for the motives of the advocates of total abstinence, the principle on which they rest is a fallacy; and all conduct resting on a fallacious principle is necessarily insecure. Moreover, we are strongly inclined 
to believe that a large portion of the reputed good ascribed to their exertions has resulted from the dissemination among the thoughtful and intelligent of the working classes, who are not themselves abstainers, of information relating to the evils, moral and economical, that flow from excess.

There is not much to detain us in a short paper on influenza, and we then come to a series on typhus, enteric, and scarlet fevers. In regard to the typhus and enteric fevers, Dr. Gairdner's chief object is to establish the truth of Dr. Jenner's views as to the perfect distinctness of the two diseases-an object which he has prosecuted with great acuteness and industry. And if Dr. Jenner's field of observation has been more extensive, the comparatively narrow bounds within which our author's investigations have been restricted afford perhaps a more favourable opportunity for an exhaustive inquiry into all the circumstances connected with the origin and consequences of separate groups of the two diseases, than can be accomplished in the much wider area over which Dr. Jenner's observations extend. Dr. Gairdner has not limited himself to the consideration of the cases that have been brought under his notice in his hospital practice. He seems to have sought out and examined carefinlly and candidly all the cases of reputed fever he could ascertain to exist in Edinburgh, and traced laboriously their origin and their results; and the upshot of his inquiries is that in no case has he been able to trace enteric fever to the contagion of typhus or the reverse. Groups of the former disease and successive cases of the other, he has found to exist at the same time in different localities, but in no instance was there ever any substitution or interchange of the two diseases among the respective groups of each. Such a statement from so trustworthy an authority is donbtless important. But an objector might remark that, after all, Dr. Gairdner's evidence is little more than negative, by which it is not very easy to prove a positive opinion; and that from the very interesting and satisfactory diminution of fever in Edinburgh during the last twelve years-a decrease all the more gratifying that we cannot avoid associating it with the better feeding, better clothing, and increased comfort of the poorer classes. Dr. Gairdner's numbers are small enough to warrant a very determinate conclusion. We must observe, too, that Dr. Gairdner founds his diagnosis exclusively on the appearance of the two eruptions of typhus and typhoïd fevers, giving up all idea of distinguishing them by their symptoms. Referring to our own experience, we think we have seen cases absolutely typical, so far as symptoms go, of enteric fever which have presented before their termination all the characteristic appearances of malignant typhus; and we state this the more confidently that a precisely similar case was mentioned to us a very short time ago by a highly-accomplished physician of great experience both in hospital and general practice. However painful, then, it may be to have established opinions unsettled, we can scarce regret that the question of the identity or non-identity of typhus and enteric fever has been reopened by Dr. Henry Kennedy, of Dublin. One of the most valuable and interesting features of Dr. Gairdner's work is its verisimilitude. Reading it is 
like listening to the commentaries of an able and skilful teacher, on cases that we actually see and watch. But the close application of his remarks to cases immediately under his superintendence, and his obvious reluctance to swell his book by general dissertations, sometimes makes us regret that he had not entered a little more fully into the general history and treatment of the diseases he considers. The epidemic scarlatina which Dr. Gairdner describes has evidently been mild. Still scarlatina is one of the most formidable diseases which occur in this island. His remarks on the treatment are extremely judicious, but there is prodigious diversity in the appearances it presents and the course that it runs; and the practitioner, while he avoids the "nimia diligentia" of Sydenham, caunot too carefully watch the progress of the disease, or be too guarded in his prognosis, till every symptom of illness is conclusively at an end. Dr. Gairdner cautions his pupils against being too much alarmed by the occurrence of delirium in this disease, because the course of the fever being short, this symptom will probably subside when the crisis of the fever is over. Speaking generally, he is right; unquestionably so, if the delirium is mild or only partial, and not always persistent or complete. But there is a delirium of a turbulent character, and accompanied with excessive restlessness, which we deem most serious. When it occurs, as we have generally seen it do, on the fourth or fifth day-mostly the fourth-when the little patient picks, or rather tears, at the lips and tongue, or moves the hands before the eyes with a kind of spinning motion, we have repeatedly, even in cases that previously seemed tolerably mild, seen it prove fatal in less than forty-eight hours. We would suggest to those of our brethren who have numerous opportunities of post-mortem examination, to look for any evidences that may exist of congestion or inflammatory exudation in the inferior surfaces of the brain, in the striated bodies, or optic thalami. Where the pulse will bear it, which often, however, is not the case, we have sometimes seen benefit from very cautious leeching
at the back of the neck.

A very good paper on cholera (malignant) follows the one we have been adverting to. It presents an excellent summary of what may be considered as established facts, especially as regards the Edinburgh epidemics, in the history of that terrible disease, without entering, however, into the vexed question of its contagious or noncontagious nature. We need not dwell on the pathological statements of this paper. The inferences deducible from them are almost entirely of a negative character. But to us, as to Dr. Gairdner, it appears pretty well made out that the characteristic symptoms of the collapse from the bowels, by which the serum, with the salts it contains, as well as albumen, are rapidly drained off from the blood. A great deal no doubt remains behind this, supposing it true, respecting the primary effects as well as the nature of the poison which produces the disease. But if this be the order of consecution so far, at least something is at least compare are able partially to account for the reported success, at least comparatively speaking, as stated in Mr. Ross's tables, of a 
treatment which seems to depend on the restoration of the abstracted fluid by the administration of cold water or of ice, and by maintaining the body throughout in contact with fluid media or aqueous vapoura treatment which seems, in addition, germane to the feelings of the patient.

The paper on delirium tremens is very sensible, and probably expresses the sentiments that have been growing among the more thoughtful of the profession for a long time-a total disbelief in the absolute and specific efficacy of opium and alcoholic stimulants in this disease, and a disposition, in certain cases at least, to trust to the gradual elimination of the poison by the efforts of nature, aided by such appliances as the particular symptoms of each individual case may suggest. If the statements brought forward regarding the treatment of delirium tremens by digitalis be corroborated by further experience, and if the physiological effects of the medicine are not exhibited when it is given in half-ounce doses, but are produced, even during the course of delirium tremens, when the quantity is diminished, as has been stated, it is a fact which, to say the least of it, opens up some very extraordinary topics of speculation.

We now draw our remarks on Dr. Gairdner to a close, but we cannot do so without characterizing, though we have not space to analyse, his various papers on diseases of the chest and their physical diagnosis. Highly as we estimate those we have so imperfectly gone over, we do not know but what those in the latter part of the volume are more valuable still. Certainly they are in no respect inferior, to say the very least, displaying throughout a calm, sagacious, and unprejudiced spirit of investigation, and a truly judicial temper in estimating the value of signs and the efficacy of remedies. There is much ingenuity and acuteness in the remarks which he makes on the cases of pneumothorax with regard especially to its diagnostic signs; while the whole discussion of pleuritic effusions is extremely temperate, rational, and fair, especially with respect to the evacuation of pleuritic effusions by puncture of the thoracic parietes. Dr. Gairdner does not appear to be a zealous advocate of this operation in acute pleurisy, while he by no means peremptorily forbids it in all cases, stating, however, that only twice in eleven years of extensive practice has he felt called upon to perform it. In the operation he has always employed the exhausting syringe introduced by Dr. Bowditch, of Boston, U.S., since he first became acquainted with that instrument.

The chapter on cardiac murmurs is an exceedingly interesting and useful one, more complete and exhaustive than most of the other papers in the volume. We regret the less our inability to give an account of it, because it will well repay attentive perusal, and indeed cannot be read with the full profit that may be derived from it otherwise than in extenso, and with the aid of the accompanying diagrams. Notwithstanding all that has been written on the subject, it is a valuable addition to the literature of auscultation." It is, however, the clear exposition that is given of the relation that subsists between

* To this subject, as treated by Dr. Gairdner, we alluded at p. 259 of the 59th number of our Review. 
physical signs and the symptoms of disease, and the just estimate that is shown of their comparative value in the cases detailed, and the accompanying remarks, which constitute, in our judgment, the peculiar value of the whole series of papers on chest diseases, as well as that on cardiac murmurs. It cannot be doubted that in practice serious mischief has resulted from a too exclusive regard to mere physical signs, without a corresponding attention to symptoms.

The sounds heard in the chest are merely acoustic phenomena, resulting from the condition of the organs it contains, and partly of the parietes that surround them. The relation that subsists between these is so far from being altogether definite, that it often requires much thought and careful study, as Dr. Gairdner clearly shows, to decipher their true meaning and import, even with reference to the organs we are examining. Further, signs give us no information as to the pathological state of the constitution, from which chiefly our indications of treatment must be drawn. Symptoms, as is truly said by Dr. John Brown, are the mother-tongue of Nature-signs no more than a foreign language.

We take leave of Dr. Gairdner and also of Dr. Begbie, by expressing our sincere hope that we may soon meet either or both in the field of medical literature, in the cultivation of which they are so highly qualified to distinguish themselves.

\section{REVIEW IV.}

Lecture on Milk; on the Composition of Cheese, and on Practical Mistakes in Cheese-mating; Cheese Experiments ; on Poisonous Cheese. By Dr. Augustus Voelcker. (Reprinted from Vols. xxii. and xxiii. of the 'Journal of the Royal Agricultural Society of England.')

IT is very interesting to see the methods of modern science applied to the illustration and improvement of some of the oldest of the arts. Dr. Angustus Voelcker's papers on the products of the dairy are a happy example of this. The limits to which we are restricted allow us to notice only some of the results of his practical inquiries. In limine, however, we have no hesitation in stating that each of the papers of which we have prefixed the title is deserving of being carefully read and studied, and not only by the farmer but also by the medical man; by the one for his own profit; by the other for the benefit of his patients-milk, butter, and cheese being acknowledged as three of the most important articles of food, and each being subject to adulteration, so as to injuriously affect the health, and the last mentioned (cheese) even to a poisonous degree.

In treating of milk, Dr. Voelcker, after describing its ordinary qualities and composition, gives some instructive particulars of the various circumstances by which these qualities are altered or modified, and of the marked differences as to composition of the milk of different
animals.

In the instance of cow's milk, of all its ingredients, he finds the 